\title{
Timely and Reliable Packets Delivery over Internet of Vehicles (loVs) for Road Accidents Prevention: A Cross-Layer Approach
}

\author{
Elias Eze $C^{1,2}$, Sijing Zhang ${ }^{1}$, Enjie Liu ${ }^{1}$, Nweso Emmanuel $N^{2}$, Joy Eze $C^{1,2}$ \\ ${ }^{1}$ Institute for Research in Applicable Computing (IRAC), Department of Computer Science and Technology, \\ University of Bedfordshire, Luton, England. ${ }^{2}$ Computer Science Dept, Ebonyi State University Abakaliki, Nigeria. \\ \{elias.eze, joy.eze1\}@study.beds.ac.uk, nwesonwogbaga@yahoo.com, \{sijing.zhang, enjie.liu\}@beds.ac.uk
}

\begin{abstract}
With the envisioned era of Internet of Things (IoTs), all aspects of Intelligent Transportation Systems (ITS) will be connected to improve transport safety, relieve traffic congestion, reduce air pollution, enhance the comfort of transportation and significantly reduce road accidents. In IoVs, regular exchange of current position, direction, velocity, etc., enables mobile vehicles to predict an upcoming accident and alert the human drivers in time or proactively take precautionary actions to avoid the accident. The actualization of this concept requires the use of channel access protocols that can guarantee reliable and timely broadcast of safety messages. This paper investigates the application of network coding concept to increase content of every transmission and achieve improved broadcast reliability with less number of retransmission. In particular, we proposed Code Aided Retransmission-based Error Recovery (CARER) scheme, introduced an RTB/CTB handshake to overcome hidden node problem and reduce packets collision rate. In order to avoid broadcast storm problem associated with the use of RTB/CTB packet in a broadcast transmission, we developed a rebroadcasting metric used to successfully select a vehicle to rebroadcast the encoded message. The performance of CARER protocol is clearly shown with detailed theoretical analysis and further validated with simulation experiments.
\end{abstract}

Keywords - VANET, Broadcast, DSRC, MAC, XOR

\section{INTRODUCTION}

The IoTs is driving the evolution of Vehicular Ad-hoc Networks (VANETs) into the IoVs paradigm [1-2]. With IoVs paradigm, vehicles are considered smart nodes equipped with multi-sensor platform, computation units and communication technologies to provide either direct or indirect IP-based connectivity to the Internet.

Each year, road accidents lead to approximately 1.2 million deaths world over [3]. According to UK Department for Transport's June 2015 reports, vehicle traffic levels increased by 2.4 percent between 2013 and 2014 with a total of 194,477 casualties of all severities in reported road traffic accidents during 2014 alone [4]. Similarly, a recent report by WHO on road traffic deaths in selected African countries says Nigeria accounts for the highest fatalities with 33.7 percent per 100,000 population every year. It is estimated that the volume of traffic in Nigeria will increase from 8 million at present, to 40 million by 2020 [5]. Despite the large number of these fatalities, they are, in principle, avoidable through the use of ITSs and safety vehicular communication systems. Statistics have shown that over 60 percent of chain traffic accidents could be prevented if drivers are informed about an automobile accident at least $500 \mathrm{~ms}$ ahead of time [6].

IoVs also has a potential to promote e-Health services by incorporating already proposed remote and mobile patient monitoring systems (MPMSs) [7-8] into vehicular networks to monitor bio-signal data of the patients who are in a stable condition. The bio-signal data of a mobile patient (i.e., passenger) can be transferred and processed for diagnosis such as blood pressure or diabetes, and cardiac disease by monitoring sugar level, and electrocardiogram (ECG) signal, respectively. Several mobile patient monitoring solutions have been proposed to continuously monitor the status of patients even in presence of mobility [7-8]. Hence, in this study, we adopted the e-Health solution proposed in [7] and proposed a scheme that will improve transmission reliability of mobile patients' collected bio-signal data to their e-Health service provider (i.e., healthcare center).

Approaches like RTS/CTS, BRTS/BCTS, ARQ and ACK/N-ACK [1,9] has been adopted to ensure reliability. However, these approaches can only work efficiently in a unicast communication as opposed to IoVs where traffic safety depends on broadcast communication techniques. Hence, relying on traditional error recovery mechanisms to ensure reliability in vehicular networks would only worsen the problem [1].

Many results have also been published on retransmission-based error recovery approaches [10-11] for VANETs. However, retransmission attempts increase network overhead and after a certain number of repeats may lead to excess channel congestion. This paper investigates the possibility of reducing the number of retransmissions while increasing the content and efficiency of each retransmission through network coding [12], significant reduction of access contention and excess signaling overhead by selecting a relaying vehicle with the aid of a node selection metric, $\eta$ to rebroadcast the encoded messages.

The main contributions addressed in this paper include: 1) Propose a novel CARER protocol which aims to guarantee timely transmission and satisfy quality of service (QoS) demands of the encoded packets through network coding by taking into consideration the special features of vehicular networks like high mobility, 
This article has been accepted for publication in a future issue of this journal, but has not been fully edited.

Content may change prior to final publication in an issue of the journal. To cite the paper please use the doi provided on the Digital Library page.

dynamic topology, channel conditions, etc; 2) Develop a rebroadcasting metric $\eta$ which is used to select the relaying vehicle to rebroadcast the encoded packets to enable the nodes outside the radio coverage of the source node to receive the encoded packets, 3) Derive an analytical model to quantify packet loss recovery, collision, access delay, and delivery rate, and 4) Evaluate the performance of CARER against a reference protocol.

The remaining part of the paper is arranged as follows: the literature review is presented in Section II. Section III discusses the system model, and presents a detailed analysis of the proposed scheme. The simulation setup, analytical and simulation results are presented in Section IV, while Section V concludes the paper.

\section{LITERATURE REVIEW}

There are already several research solutions proposed to leverage on the ubiquitous feature of IoTs to provide out-of-hospital follow-up services [7-8]. The study conducted by Yang et al. [13] demonstrates a possible way of improving the validity of life-logging physical activity data in an IoTs environment through wearable devices. The authors projected a distribution of irregular uncertainty by defining a walking speed related score called Daily Activity in Physical Space (DAPS). Additionally, an ellipse fitting model based validity improvement method for reducing uncertainties of life-logging physical activity measures in an IoTs environment was presented. Other IoTs aided healthcare studies are reported in [14-18].

Despite the efficiency of these proposed solutions, none of the works has actually focussed on the challenges of reliable delivery of the monitored patients' data to health centers for urgent processing and prescription. Given the potential life-threatening situations associated with the data acquired through MPMSs, guaranteeing the reliability of message (i.e., bio-signal data) delivery from moving vehicles to healthcare center to ensure urgent intervention from health care professionals as and when required is the most crucial requirement of ehealth systems. Additionally, the proposed protocol should be capable of delivering the packets carrying vital signs like passenger's (or mobile patients) bio-signal data within a guaranteed time determined by the level of emergency of the concerned patient.

One of the existing works that has actually tried to tackle the challenge of data delivery reliability in MPMSs is the study conducted by Varshney and Sneha [19]. The authors investigated on how to provide support for reliable data transmission from wireless patient monitoring to health care professionals by proposing several protocols for power management of devices, assisted power control, and sleep strategy. Other related works include an e-hospital-wide MPMS [20], wireless monitoring of physiological data for heart failure patients [21], wireless MPMS [22], long-term MPMS via wireless wearable devices [23], wireless real-time electroencephalography (EEG) epilepsy monitoring system [24], and reliability in wireless MPMS [25].

For mobile patients (i.e. passengers in a mobile vehicles) with cardiac diseases, ECG signal can be monitored and transmitted wirelessly to the healthcare center. However, none of them has actually taken into consideration the peculiar application requirements and communication constraints associated with IoVs (or VANETs). Furthermore, none of the above solutions consider the benefits of applying network coding to guarantee the reliability of the transmitted bio-signal data. Hence, our proposed CARER protocol is designed with these considerations in mind to guarantee timely and reliable transmission of these monitored bio-signals and other vehicular safety messages, while satisfying QoS requirements. Interestingly, some authors have worked on improving data transmission reliability through the application of network coding technique. Originally, Li et al [26] followed the pioneering work of Ahlswede et al [12] on the concept of network coding, to show that linear codes can be used to achieve the maximum capacity bounds for multicast traffic. Lun et al [27] built on this foundation to study network coding with omnidirectional antennae and showed that the challenge of minimizing the communication cost can be expressed as a linear program that can be solved through a distributed approach.

With the manifold benefits of network coding, the main challenge is how to improve reliability of one-hop broadcast communication using this concept. Nguyen et al [28] proposed scheme that can efficiently recover lost packets in one-hop wireless broadcast retransmissions through network coding. Their schemes are not applicable in VANETs because the packets that are XORed for retransmission are generated from a single node over a period of time. In VANETs, all nodes broadcast their packets containing their real-time status where the most recent packets supersede the previous packets due to change in position and other kinematic data. Hence, only currently broadcasted packets are encoded for retransmission as opposed to the scheme referred to in [28].

Recent investigation by Wang and Hassan [29] studied a different type of repetition-based error recovery techniques. The study presented in [28] falls in the category of our proposed protocol given that they applied network coding to increase the content of each retransmission attempt, but they lack the contributions of our proposed novel protocol as itemized in Section I. Additionally, CARER protocol presents an in-depth analytical model of a loss recovery scheme that is not limited to merely two packets but can combine $n$-packets received from other vehicles as well as the self-generated native packets. 
This article has been accepted for publication in a future issue of this journal, but has not been fully edited.

Content may change prior to final publication in an issue of the journal. To cite the paper please use the doi provided on the Digital Library page.

\section{PROPOSED CARER PROTOCOL}

We consider a highway of 4 lanes with half of the lanes used for nodes moving to one direction and the other half used for nodes moving to the opposite direction. The vehicles maintain directional velocity which is randomly distributed among a discrete set $V=\{(30+10 * k) m / s, \forall k \in[0,5]\}$. The nodes are equipped with a GPS which is used to acquire position information, moving direction and velocity, and a half-duplex transceiver for wireless communication. The CSMA/CA-based IEEE 802.11e MAC is used for channel access and service differentiation with some modification. The traditional three-way handshake of CSMA/CA-based MAC is adopted with certain modifications by using request-to-broadcast and clear-to-broadcast (RTB/CTB) packet exchange before encoded messages are transmitted. Additionally, CARER protocol uses a location-aware algorithm (LAA) [30], and the metric $\eta$ specifically designed for vehicular communications to select one vehicle to rebroadcast the encoded message along the intended propagation direction.

\section{A. Safety (or High Priority) Message Coding}

Each mobile node performs an exclusive OR (XOR) operation over its own-generated raw packets and any other packets contained in $\rho$ (i.e. packets received from other vehicles in $T \mathrm{~ms}$ ). Upon successful packets encoding, nodes $(i+1)$ and $(i-1)$ retransmit the encoded versions of the packets $[i \oplus(i+1)]$ and $[(i-1) \oplus$ $i]$ for $(m+1)$ times, respectively to enable every vehicle within their vicinity to recover any packet(s) they may have lost. Packet recovery probability (PRP), defined as the probability that a raw packet is lost but recovered with CARER scheme after $m$ retransmission attempts. We used analytical study to derive PRP as a function of the link error probability. Let $P_{l}(C A R E R)$ be the loss probability of CARER protocol. Then, we have

$$
P_{l}(\text { CARER })=\epsilon_{i}\left[\left(1-\alpha_{i}\right)\left(1-\beta_{i}\right)\right]
$$

where $\epsilon_{i}$ represents the error probability, $\alpha_{i}$ and $\beta_{i}$ represent the probability that node $i$ can successfully recover raw packet $i$ after successfully decoding the encoded retransmitted packets $[(i-1) \oplus i]$ and $[i \oplus$ $(i+1)$ ], respectively. $P_{l}(C)$ implies that: 1$)$ node $i$ lost the raw packet $i$; $)$ node $(i-1)$ cannot recover raw packet $i$ from the encoded packet retransmission $[(i-1) \oplus i]$; and (3) node $(i+1)$ cannot recover raw packet $i$ from the encoded packet retransmission $[i \oplus(i+1)]$. Considering that node $(i-1)$ repeats the encoded packets $[(i-n) \oplus n i]$ for a total of $(m+1)$ times before all the vehicles within the radio communication range of vehicle $(i-1)$ are able to successfully recover their lost packet(s), it follows that $\alpha_{i}$ is the probability that at least a single packet was received out of the $(m+1)$ retransmitted encoded packet $[(i-n) \oplus n i]$ by vehicle $(i-1)$. Hence, the encoded retransmission $[(i-n) \oplus n i]$ by vehicle $(i-1)$ can be recovered as shown in the formula:

$$
\alpha_{i}=\mu_{i}\left(\frac{\left(1-\epsilon_{(i-n)}^{(m+1)}\right)}{1-\left(1-\epsilon_{(i+n)}\right)}\right)
$$

where $\mu_{i}$ represents the probability that vehicle $(i-1)$ 's retransmitted encoded packets, $[(i-n) \oplus n i]$ can be decoded by node $i$ when successfully received. Since vehicle $(i-1),(i-2), \ldots,(i-n)$ must have at least one of $(i-1),(i-2), \ldots,(i-n)$ raw packets to be able to decode any of the encoded packets such as $[(i-1) \oplus$ $i],[(i-2) \oplus 2 i], \ldots,[(i-n) \oplus n i]$ retransmissions, $\mu_{i}$ as well means that vehicle $(i-n)$ already has at least one of the $[(i-1) \oplus i],[(i-2) \oplus 2 i], \ldots,[(i-n) \oplus n i]$ packets and therefore can decode the retransmissions of the encoded packets. So that we can now have:

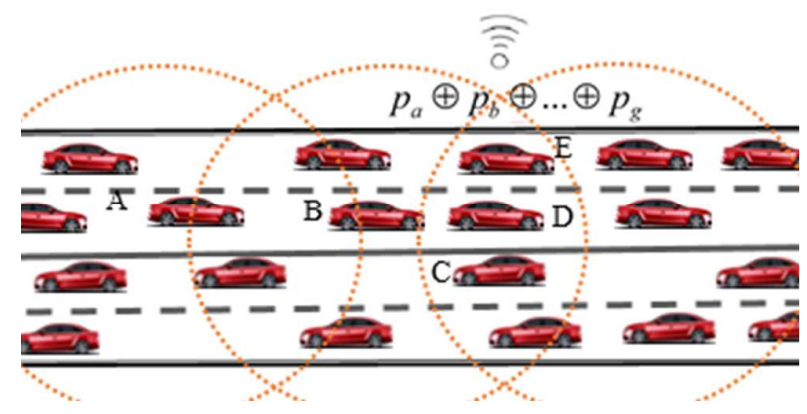

Fig. 1: CARER Protocol: Vehicle A successfully select $D$ to retransmit the encoded message so as to enable the vehicles outside one-hop broadcast range of vehicle $A$ to receive the encoded message. 
This article has been accepted for publication in a future issue of this journal, but has not been fully edited.

Content may change prior to final publication in an issue of the journal. To cite the paper please use the doi provided on the Digital Library page.

$$
\mu_{i}=1-\epsilon_{(i+n)}
$$

where $\epsilon_{i-n}$ represent the loss probability of the encoded packets $[(i-n) \oplus n i]$. Hence, eq. (2) can now be rewritten as:

$$
\alpha_{i}=\left(\frac{\left(1-\epsilon_{(i-n)}\right)\left(1-\epsilon_{(i-n)}^{(m+1)}\right)}{1-\left(1-\epsilon_{(i-n)}\right)}\right)
$$

With the encoded messages, the area of interest for retransmission is the immediate transmission range of vehicle $i$ which is successfully selected to rebroadcast the message (see Fig. 1). From eq. (4), it is observed that $\alpha_{i}$ strongly depends on $\alpha_{(i-1)}, \alpha_{(i-2)}, \alpha_{(i-3)}$, and so on, till $\alpha_{(i-n)}$. However, it is assumed that the difference between $(i-1),(i-2), \ldots,(i-n)$ is trivial and insignificant. Hence, substituting $(i-n)$ with $(i-1)$ in (4), gives a linear equation of $\alpha_{i}$ which can be solved as

$$
\alpha_{i}=\left(\frac{\left(1-\epsilon_{(i-1)}\right)\left(1-\epsilon_{(i-1)}^{(m+1)}\right)}{1-\left(1-\epsilon_{(i-1)}\right)}\right)
$$

Following the above analysis for $[(i-n) \oplus n i]$, encoded message $[n i \oplus(i+n)]$ retransmission by vehicle $(i-n)$ can be decoded by using the formula:

$$
\beta_{i}=X_{i}\left(\frac{\left(1-\epsilon_{(i+n)}^{(m+1)}\right)}{1-\left(1-\epsilon_{(i+n)}\right)}\right)
$$

where $X_{i}$ represents the probability that vehicle $(i+1)$ 's retransmitted XORed packets, $[n i \oplus(i+n)]$ can be decoded by node $i$ when successfully received. Again, following the steps that lead to the derivation of eq. (3), we have

$$
X_{i}=1-\epsilon_{(i+n)}
$$

Hence, putting eq. (7) and substituting $(i-n)$ with $(i-1)$ in eq. (6) gives a linear equation of $\beta_{i}$ which can be solved as

$$
\beta_{i}=\left(\frac{\left(1-\epsilon_{(i+1)}\right)\left(1-\epsilon_{i+1}^{m+1}\right)}{1-\left(1-\epsilon_{(i+1)}\right)}\right)
$$

Finally, putting eq. (6) and (8) into (1), $P_{l}(C A R E R)$ can be rewritten as

$$
P_{l}(\text { CRER })=\epsilon_{i}\left(\left(1-\frac{\left[\left(1-\epsilon_{(i-1)}\right)\left(1-\epsilon_{(i-1)}^{(m+1)}\right)\right]}{1-\left(1-\epsilon_{(i-1)}\right)}\right) \times\left(1-\frac{\left[\left(1-\epsilon_{(i+1)}\right)\left(1-\epsilon_{(i+1)}^{(m+1)}\right)\right]}{1-\left(1-\epsilon_{(i+1)}\right)}\right)\right)
$$

Once more, with reference to the assumed triviality of the difference between vehicles $(i-1),(i-2), \ldots,(i-$ $n)$ and $(i+1),(i+2), \ldots,(i+n)$ with respect to vehicle $i$, both the loss probability of the encoded safety messages $(i-1)$, (i.e. $\in_{(i-1)}$ ), and $(i+1)$, (i.e. $\in_{(i+1)}$ ) can be represented as $\in_{i}$. Hence, eq. (9) can be simplified and expressed as a functions of $m$ and $\epsilon_{i}$, resulting in

$$
P_{l}(\text { CRER })=\epsilon_{i}\left(\left(1-\frac{\left[\left(1-\epsilon_{i}\right)\left(1-\epsilon_{i}^{(m+1)}\right)\right]}{1-\left(1-\epsilon_{i}\right)}\right) \times\left(1-\frac{\left[\left(1-\epsilon_{i}\right)\left(1-\epsilon_{i}^{(m+1)}\right)\right]}{1-\left(1-\epsilon_{i}\right)}\right)\right) .
$$

Then, the recovery probability $\left(P_{r}\right)$ of CARER scheme will be given as:

$$
\begin{gathered}
P_{r}(\text { CARER })=1-P_{l}(\text { CARER }) \\
=1-\left[\epsilon_{i}\left(\left(1-\frac{\left[\left(1-\epsilon_{i}\right)\left(1-\epsilon_{i}^{(m+1)}\right)\right]}{1-\left(1-\epsilon_{i}\right)}\right) \times\left(1-\frac{\left[\left(1-\epsilon_{i}\right)\left(1-\epsilon_{i}^{(m+1)}\right)\right]}{1-\left(1-\epsilon_{i}\right)}\right)\right)\right] .
\end{gathered}
$$


This article has been accepted for publication in a future issue of this journal, but has not been fully edited. Content may change prior to final publication in an issue of the journal. To cite the paper please use the doi provided on the Digital Library page.

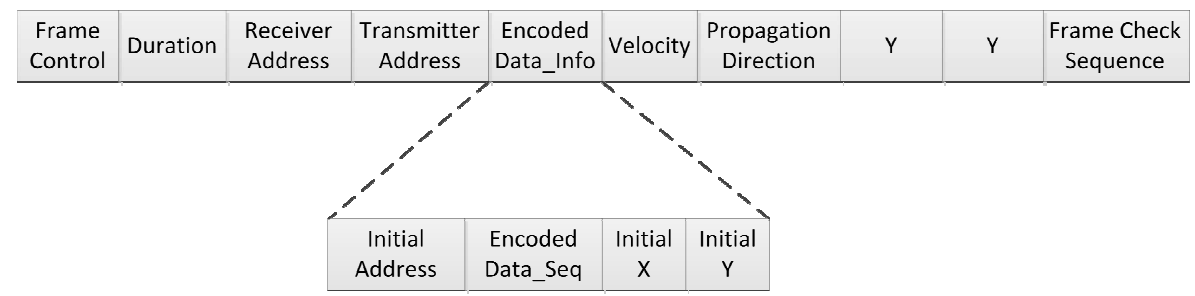

Fig. 2: An RTB frame structure

\section{B. RTB/CTB Handshake}

In order to overcome the hidden node problem and reduce packets collision, sender nodes engage in $\mathrm{RTB} / \mathrm{CTB}$ handshake with the recipients within its radio transmission range. If the furthest vehicle away can be selected with RTB/CTB packets, then other nodes in between can overhear the transmission as well. The sender adheres to all the rules of CSMA/CA transmission associated with IEEE 802.11 while attempting to broadcast an RTB packet.

Fig. 2 shows the structure of an RTB frame with an additional five fields (Encoded Data_Info, Velocity, Propagation Direction, $X$ and $Y$ ) as opposed to conventional RTS frame. The Encoded Data_Info field holds the information about the source node that initially broadcasted the encoded packets. The other fields include Velocity which is the relative moving velocity of the source node, Propagation Direction which is the encoded message desired propagation direction, while $X$ and $Y$ represent the coordinates of the transmitter. Encoded Data_Info field, in turn, contains 1) the address of the source node Initial Address, 2) the initial coordinates (Initial $X$ and Initial $Y$ ) of the source node, and 3) the encoded message's sequence number, Encoded Data_Seq.

The source node with the encoded message for transmission will first broadcast a RTB frame and obeys the CSMA/CA-based MAC procedure by starting a retransmission timer with value set as $T_{R T B_{-} r}=T_{D I F S}+T_{R T B}+$ $T_{C T B}$, where $T_{D I F S}$ represents the time duration of a Distributed coordination function Inter-Frame Space (DIFS), $T_{R T B}$ and $T_{C T B}$ represent the transmission time durations of an RTB and a CTB frame respectively. In the event of collision with no CTB response from an eligible candidate within $T_{R T B_{r} r}$ time duration, the source vehicle immediately contends for channel access to rebroadcast an RTB message until a CTB message is successfully received. With the help of the broadcast node s' information contained in the Propagation Direction fields of an RTB frame, any vehicle that overhears the RTB frame but not moving in the desired propagation direction of the encoded message will not respond with a CTB message but will set its Network Allocation Vector (NAV) and defer its own transmissions, accordingly.

On the contrary, if a vehicle receives an RTB frame, it will use the LAA to check whether it is eligible for replying a CTB frame based on the position information contained in the received RTB frame. This is possible since each vehicle broadcasts its periodic status message which contains its address, position, moving speed, and direction of movement. Hence, using the LAA after receiving an RTB frame, a node is able to know if there are other nodes within the radio transmission range of the source node ahead of itself. However, if the node's position is between the source vehicle and other vehicles in radio coverage of the sender vehicle, it will not reply with a CTB frame because there is no significant distance gain along the encoded message desired propagation direction. Hence, the node's NAV will be updated in accordance with the duration field in the received RTB frame. Otherwise, a back-off timer will be started to reply with a CTB frame, after which the node keeps sensing the channel in the meantime to receive the encoded message for a rebroadcast. For instance, in Fig. 1, node $B$ will not reply with a CTB frame after learning that there are other vehicles with higher distance gain using the LAA algorithm but rather updates its NAV. Likewise, vehicle $C$ will also update its NAV after receiving the RTB since the direction information contained in the direction field of the RTB frame shows a different intended propagation direction.

If there exist only node $i$ with the highest distance gain within the transmission coverage and in the desired propagation direction of the source vehicle, then node $i$ is selected as the eligible candidate using LAA and will start a back-off timer upon receiving an RTB frame for replying with a CTB frame to the source node. However, if there exist more than one eligible station as is the case in Fig. 1 (see node $D$ and $E$ ), each eligible node will start a back-off timer to reply with a CTB frame with the help of the following metrics: 1) the distance gain $\Delta d$, between node $i$ and the source node; 2 ) the received signal-to-noise-ratio (SNR) and packet error rate (PER), $e$, which can be estimated from the received RTB frame, and 3) the relative velocity between node $i$ and the source 
This article has been accepted for publication in a future issue of this journal, but has not been fully edited.

Content may change prior to final publication in an issue of the journal. To cite the paper please use the doi provided on the Digital Library page.

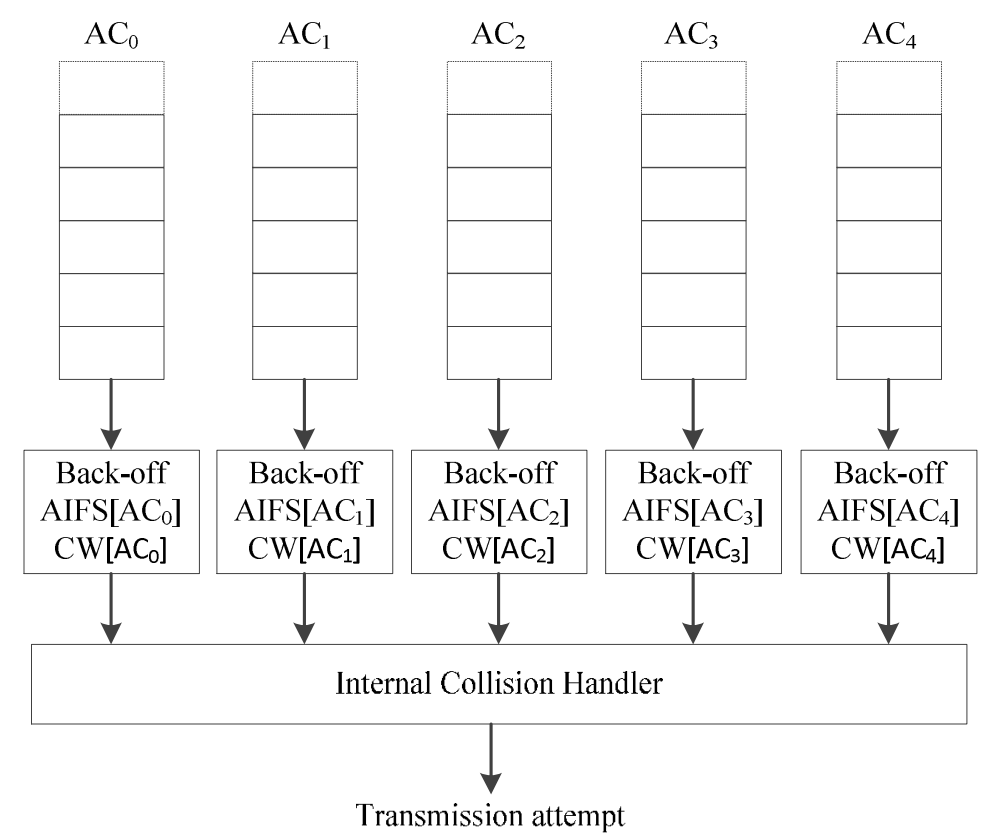

Fig. 3: The refined 802.11e MAC with five ACs

TABLe I. VALue of PARAMETERS For DifFerent AC SERVices

\begin{tabular}{|c|c|c|c|c|c|}
\hline \hline AC & CWmin & CWmax & AIFSN & PF & Wait-time \\
\hline \hline $\mathbf{0}$ & $a C W_{\min }$ & $a C W_{\max }$ & 9 & 2 & $264 \mu \mathrm{s}$ \\
\hline $\mathbf{1}$ & $a C W_{\min }$ & $a C W_{\min }$ & 6 & 2 & $152 \mu \mathrm{s}$ \\
\hline $\mathbf{2}$ & {$\left[a C W_{\min }+1 / 2\right]-1$} & {$\left[a C W_{\min }+1 / 2\right]-1$} & 3 & 2 & $72 \mu \mathrm{s}$ \\
\hline $\mathbf{3}$ & {$\left[a C W_{\min }+1 / 4\right]-1$} & {$\left[a C W_{\min }+1 / 2\right]-1$} & 2 & 2 & $56 \mu \mathrm{s}$ \\
\hline $\mathbf{4}$ & {$\left[a C W_{\min }+1 / 4\right]-1$} & {$\left[a C W_{\min }+1 / 2\right]-1$} & 2 & 1 & $56 \mu \mathrm{s}$ \\
\hline
\end{tabular}

node. Based on these criteria, the rebroadcasting metric $\eta$ is evaluated and used to determine and select the most suitably qualified candidate to rebroadcast the encoded packets. The metric is given by

$$
\eta=\frac{e}{E_{\max }}+\left[\frac{\Delta v}{V_{P}}+\left(1-\frac{\Delta d}{R_{T}}\right)\right]
$$

where $\Delta d$ is the encoded packet transmission distance which is given by the difference between the location of the source vehicle and the recipient, $R_{T}$ is the maximum IEEE $802.11 \mathrm{p}$ radio signal transmission range [31], $e$ is the PER of the encoded packet which is determined based on the calculated SNR, $E_{\text {max }}$ is the maximum acceptable PER, $\Delta v$ and $V_{P}$ is the relative and maximum velocity, respectively.

CARER protocol requires the selected vehicle to reply the source vehicle with a CTB frame within the DIFS interval in order to prevent interruption of an RTB/CTB handshake mechanism between itself and the selected node from other flows. Finally, the station with the minimum value of $\eta$ will reply the source node with a CTB frame first and, then becomes the selected node to rebroadcast the encoded message. Eventually, other vehicles will end up updating their NAVs, accordingly, whenever they receive or overhear other RTB/CTB frames.

By applying the concept of mini-slot [1], the DIFS interval is further divide into a number of mini-slots. Using CARER protocol, vehicles start a timer in terms of mini-slots to enable them to contend for medium access. The total number of mini-slots $M S_{n}$ can be calculated as

$$
M S_{n}=\left|\frac{T_{D I F S}}{\Gamma}\right|
$$

and $\Gamma$ denotes the length of a mini-slot which is given by 
This article has been accepted for publication in a future issue of this journal, but has not been fully edited.

Content may change prior to final publication in an issue of the journal. To cite the paper please use the doi provided on the Digital Library page.

$$
\Gamma=T_{\Delta}+2 \cdot \ell
$$

where $T_{\Delta}$ represents the total duration it takes a transceiver to switch between receiving and transmitting modes, and $\ell$ denotes the average propagation delay of the channel within the transmission range, $T_{r}$. The rebroadcasting metric $\eta$ is then mapped to $M S_{n}$ by dividing $\eta$ into $M S_{n}$ different segments, and each partition is given by

$$
\mathcal{P}_{0}=\left\lfloor\frac{\eta}{M S_{n}}\right\rfloor
$$

Upon successful evaluation of the rebroadcasting metric $\eta$, each eligible rebroadcasting vehicle sets its backoff timer to $\mathrm{k}$ mini-slots, provided its $\eta$ falls within $\left\{\eta_{\min }+\left((\mathrm{k}-1) \cdot \mathcal{P}_{0}\right),\left(\eta_{\min }+\left(\mathrm{k} \cdot \mathcal{P}_{0}\right)\right)\right\}$, where $\mathrm{k}$ falls between the range of 1 to $M S_{n}$ (i.e., $\mathrm{k} \in\left[1, M S_{n}\right]$ ). Finally, the vehicle that has the minimum value of $\eta$ will eventually reply the source node with a CTB frame first, and will be selected as the current rebroadcasting station. In order to determine the received signal power, we adopt the Friis free-space model in our theoretical analysis. In line with Proakis [32], the bit error rate (BER) of the encoded messages over an AWGN channel with binary phase-shift keying modulation is taken as $Q\left(\sqrt{\left(2 \varepsilon_{b} / N_{0}\right)}\right)=Q\left(\sqrt{\left(2 P_{r} / r_{b} N_{0}\right)}\right)$, where $Q(x)=$ $(1 / \sqrt{2 \pi}) \int_{x}^{\infty} e^{-t^{2} / 2 d t}, N_{0}$ is the noise power spectral density, $\mathcal{E}_{b}$ is the received energy per bit, $r_{b}$ is the basic rate and $P_{r}$ is the received power. In [33], it is given that $e=1-\left(1-Q\left(\sqrt{\left(2 P_{r} / r_{b} N_{0}\right)}\right)\right)^{L}=1-$ $(1-Q(I / \Delta d))^{L}$, where $I=\sqrt{\left(2 P_{t} G_{t} G_{r}\left(c / f_{c}\right)^{2}\right) /\left(r_{b} N_{0}(4 \pi)^{2}\right)}, P_{t}$ is the transmission power, $G_{t}$ is the transmitter antenna gain, $G_{r}$ is the receiver antenna gain, $c$ is the measured speed of light, and $f_{c}$ is the carrier frequency. Using the definition of PER $e$ as given above, then eq. (12) can be rewritten as

$$
\eta=\left[1-\left(1-Q\left(\frac{I}{\Delta d}\right)\right)\right]^{L}\left(E_{\max }\right)^{-1}+\left[\frac{\Delta v}{V_{P}}+\left(1-\frac{\Delta d}{R}\right)\right]
$$

Therefore, $\eta$ is a function of $\Delta v, V_{P}$, and $\Delta d$ once the values of the parameters such as $I, L$ and $R$ are obtained. The minimum and maximum values of $\eta$ are denoted by $\eta_{\min }$ and $\eta_{\max }$, respectively. Consequently, it shows that the selection of mini-slots for network channel access contention solely depends on difference in distance as well as relative velocity to the source vehicle.

\section{Safety Message Requirement Satisfaction}

CARER uses a multi-channel concept which accommodates both safety-related and infotainment messages and offers priority to different messages by the use of different Access Classes (ACs) with different channel access settings, which enables safety messages to be transmitted timely and reliably, even in a dense vehicular network environment. Therefore, to ensure provision of guaranteed acceptable minimum delay to safety-related messages, we adopted and refined the widely used priority-based Enhanced Distribution Channel Access 802.11e (EDCA) scheme for service differentiation and sub-divided all the services into five (5) different classes (see Fig. 3).

The QoS demands of the different ACs are differentiated by assigning different parameters such as AIFS values, $\mathrm{CW}$ sizes, and TXOP limits. For encoded safety messages, smaller values of AIFS/CW are assigned to increase the probability of winning the channel access contention (see Table I). Similarly, assigning larger TXOP limit, elongates the channel holding periods of the vehicle that won the channel access contention. Each AC maintains a different Arbitration Inter-Frame Space Number (AIFSN) to ensure that high priority class has less wait time for channel utilization. CARER uses the same settings of AIFS and contention window (CW) specified in IEEE 802.11e as shown in Eq. (17) and (18) below;

$$
\begin{gathered}
A I F S[A C]=T_{S I F S}+\operatorname{AIFSN}[A C] \cdot \text { aSlotTime } \\
C W[A C]=\min \left\{(C W[A C]+1) P F[A C], C W_{\max }[A C]\right\}
\end{gathered}
$$

where AIFSN (i.e. $\operatorname{AIFSN}_{[A C]} \geq 2$ ) is a positive integer, aSlotTime denotes the duration of a time-slot, and PF is the persistence factor, which is set to 1 and 2 for encoded safety and non-safety messages, respectively. From Eq. (17) and (18), it can be noticed that as the values of the minimum CW and AIFSN decrease, the priority of the corresponding class increases, accordingly. Consequently, vehicle $i$ usually selects a back-off counter from the minimum $\mathrm{CW}$ for encoded safety message transmission, and doubles the $\mathrm{CW}$ after each unsuccessful 
This article has been accepted for publication in a future issue of this journal, but has not been fully edited.

Content may change prior to final publication in an issue of the journal. To cite the paper please use the doi provided on the Digital Library page.

retransmission attempt up to a maximum value referred to as maximum $\mathrm{CW}\left(C W_{\max }\right)$. Hence, this process guarantees that encoded safety (or high priority mobile patient's bio-signal) messages will always have the highest service priority.

\section{Resolution of CTB Packets Collision(s)}

When there are more than one vehicle with the furthest possible equal distance gain from the source vehicle, all of them may find the channel empty after receiving RTB packet and continue to send CTB packets. Since all vehicles start sending the CTB packets at the same time, their CTB packets will eventually result in collision. As a result, when the source vehicle overhears a transmission but unable to decode the CTB frame, it automatically detects the resultant collision and retransmits the RTB frame after SIFS time duration, as shown in Fig. 4(a).

However, only the eligible rebroadcasting vehicles that have sent CTB frames which lead to collision will join the collision resolution. When an eligible rebroadcasting node that has formerly replied with a CTB packet

(a)

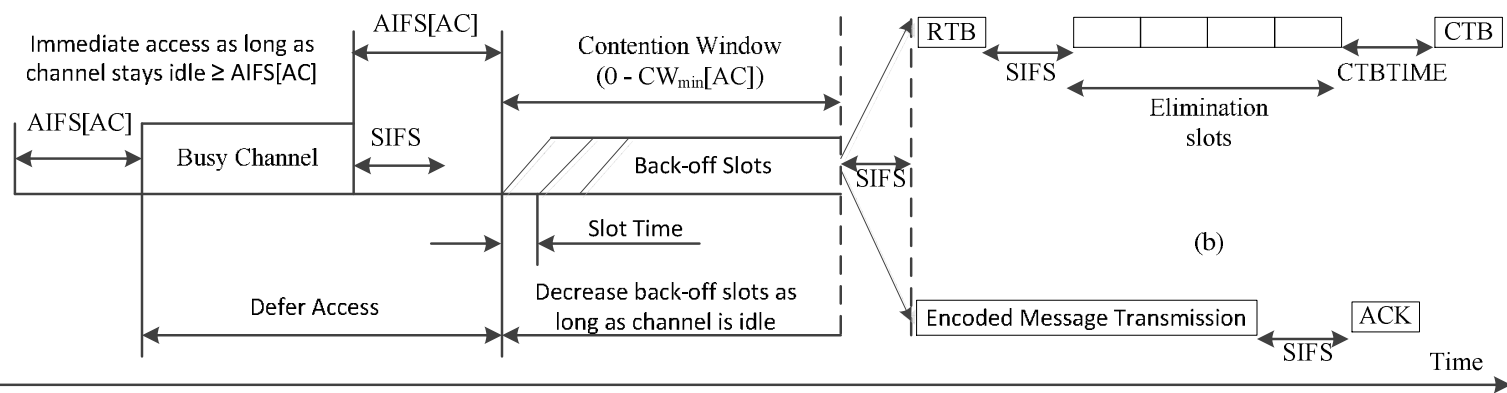

(a) When CTB packets from two or more vehicles result in collision, RTB is retransmitted again

(b) When CTB packet from the source vehicle is successfully received by a selected rebroadcasting vehicle, encoded message is transmitted

Fig. 4: Transmission sequence of packets. (a) Repeat of RTB and CTB packets. (b) Encoded messages and ACK packets.

TABLE II. REBROADCASTING VEHICLE SELECTION ALGORITHM

\begin{tabular}{|c|c|}
\hline \multicolumn{2}{|r|}{ Algorithm 1: Rebroadcasting Vehicle Selection Algorithm } \\
\hline 1: & Vehicle $i$ received an RTB short frame \\
\hline 2: & If Transmitter_address $=$ Initial_address Then \\
\hline 3: & If vehicle $i$ received the RTB short frame for the first time Then \\
\hline 4: & check Propagation_direction. \\
\hline 5: & If vehicle $i$ is moving in the intended propagation direction Then \\
\hline 6: & GoTo 1 \\
\hline 7: & Else \\
\hline 8: & set the network_allocation_vector(NAV) \\
\hline 9: & End if \\
\hline 10: & Else \\
\hline 11: & GoTo 1 \\
\hline 12: & End if \\
\hline 13: & Else \\
\hline 14: & If vehicle $i$ received the RTB short frame for the first time Then \\
\hline 15: & $\begin{array}{l}\text { If vehicle } i \text { maintains a distance gain in the intended } \\
\text { propagation direction Then }\end{array}$ \\
\hline 16: & GoTo 1 \\
\hline 17: & Else \\
\hline 18: & set the NAV \\
\hline 19: & End if \\
\hline 20: & Else \\
\hline 21: & GoTo 1 \\
\hline 22: & End if \\
\hline 23: & End if \\
\hline 24: & Compute the PER, $\eta_{\min }, \mathcal{P}_{0}$, velocity and distance gain \\
\hline 25: & $\eta \leftarrow$ vehicle $i$ to mini-slots \\
\hline 26: & Initialise the back-off timer \\
\hline 27: & GoTo 1 \\
\hline
\end{tabular}


This article has been accepted for publication in a future issue of this journal, but has not been fully edited.

Content may change prior to final publication in an issue of the journal. To cite the paper please use the doi provided on the Digital Library page.

\begin{tabular}{|l|c|}
\hline 28: & If $0<$ repeat_limit $<W_{\max }$ Then \\
\hline 29: & Compute $\mathcal{P}_{\text {repeat_limit }}=\mathcal{P}_{0} /\left[M S_{n}\right]^{\text {repeat_limit }}$ \\
\hline 30: & $\eta \leftarrow$ vehicle $i$ to mini-slots \\
\hline 31: & Initialise the back-off timer \\
\hline 32: & GoTo 1 \\
\hline 33: & Else \\
\hline 34: & Arbitrarily select a mini-slot $M S_{n}$ \\
\hline 35: & Initialise the back-off timer \\
\hline 36: & GoTo 1 \\
\hline 37: & End if \\
\hline 38: & While back-off counter $\neq 0$ Do \\
\hline 39: & If vehicle $i$ receives the CTB short frame, a \\
\hline & response from the RTB packet Then \\
\hline 40: & Stop the back-off counter \\
\hline 41: & Set the NAV \\
\hline 42: & Break \\
\hline 43: & End if \\
\hline 44: & End while \\
\hline 45: & If back-off counter $=0$ Then \\
\hline 46: & Reply with a CTB packet \\
\hline 47: & End if \\
\hline 48: & Return \\
\hline
\end{tabular}

to the source vehicle receives a retransmitted RTB packet, it will sub-divide its $\mathcal{P}_{0}$ into $M S_{n}$ segments. Hence, each of the sub-segments will be $\mathcal{P}_{1}=\mathcal{P}_{0} / M S_{n}$. The vehicle now selects a random mini-slot, starts the back-off stage and divides their $\eta$ with $\left.\left[\left|\eta_{\text {min }}+\left(\eta-\eta_{\text {min }}\right) / \mathcal{P}_{1}\right| \cdot \mathcal{P}_{0}, \eta_{\text {min }}+\left(\mid \eta-\eta_{\text {min }}\right) / \mathcal{P}_{1} \mid+1\right) \cdot \mathcal{P}_{0}\right]$ into $M S_{n}$ subsegments. The vehicle then waits for a total of $k$ mini-slots (where $k \in\left\lfloor 1, M S_{n}\right\rfloor$ ) to reply the source vehicle with a CTB packet again, only if $\left[\eta_{\text {min }}+\left|\left(\eta-\eta_{\text {min }}\right) / \mathcal{P}_{1}\right| \cdot \mathcal{P}_{0}+(k-1) \mathcal{P}_{1]} \leq \eta<\left[\eta_{\text {min }}+\left|\left(\eta-\eta_{\text {min }}\right) / \mathcal{P}_{1}\right| \cdot\right.\right.$ $\left.\mathcal{P}_{0}+\left(k \cdot \mathcal{P}_{1}\right)\right]$. This process continues iteratively until a successful CTB frame is received by the source vehicle and a rebroadcasting vehicle is finally selected or until retransmissions due to CTB packet collisions reach $W_{\max }$ times. The designed collision resolution algorithm is highly efficient for selecting a unique rebroadcasting vehicle. Below, in Table II, is the pseudo-code of the rebroadcasting vehicle selection procedures.

\section{E. Dissemination of Encoded Message and ACK Packets}

Upon successful reception of a CTB packet, the source transmits its encoded message (see Fig. 4(b)). The source vehicle also includes in the broadcasted encoded packet, the IP address of the selected rebroadcasting vehicle which had replied with a CTB short frame. Consequently, only the selected rebroadcasting vehicle whose IP address is contained in the broadcasted encoded message is responsible for sending an ACK packet to the source vehicle and rebroadcasting (i.e. forwarding) the encoded message to ensure greater transmission coverage beyond one-hop transmission of the source vehicle.

The ACK short frame when received by the source vehicle guarantees the reliability of the encoded message dissemination and delivery in the desired propagation direction. If the source vehicle does not receive the ACK packet from the selected rebroadcasting vehicle before the set ACK time-out, then the back-off procedure explained in Section III.B is followed. The steps adhered to in this back-off process are the same as those of legacy IEEE 802.11 standards family when an ACK packet is not received by the source node before a time-out.

\section{F. Encoded Message Redundancy Control}

Each vehicle maintains a list that contains the records of the recently received encoded packets within the last $T$ ms. The entries in the list include the source vehicle's address $\left(S \_A d d r\right)$ and sequence number $\left(S e q \_N u m\right)$ of the encoded packets. When vehicle $i$ receives an encoded message, it checks the list and drops the encoded message if its $S e q \_N u m$ and $S \_A d d r$ already exist in the list. Hence, CARER controls and prevents encoded packets redundancy by automatically deleting duplicates. Otherwise, the entries of the list will be updated, accordingly.

\section{IV.SIMULATION SETUP}

The simulation was performed using NS-2 [34] which is a well-used simulator in analysing vehicular networks, to validate our analytical model. The choice of suitable vehicular movement pattern for the experiments is significant to enable us to achieve close-to-real-life scenarios with dynamic vehicular network topology. We use a highway road network with 2000m edge length and Bonn-Motion tool [1] to generate suitable vehicular mobility model. In our simulations, there are random distribution of 200 vehicles in a two op- 


\section{IET Networks}

This article has been accepted for publication in a future issue of this journal, but has not been fully edited.

Content may change prior to final publication in an issue of the journal. To cite the paper please use the doi provided on the Digital Library page.

Table III. Value of parameters used in our simulations

\begin{tabular}{|c|l||l|l|}
\hline \hline Parameter & Value & Parameter & Value \\
\hline \hline Frequency & $5.9 \mathrm{GHz}$ & Data rate & $3 \mathrm{Mbps}$ \\
\hline Bandwidth & $10 \mathrm{MHz}$ & DIFS time & $64 \mu \mathrm{s}$ \\
\hline Modulation & BPSK & TX power & $2 \mathrm{~mW}$ \\
\hline Packet size & 512 byte & $A R$ & 37 byte \\
\hline $\boldsymbol{E}_{\max }$ & $8 \%$ & $A G$ & 17 byte \\
\hline $\boldsymbol{f}_{\boldsymbol{c}}$ & $2.4 \mathrm{G}$ & $V_{P}$ & $50 \mathrm{~m} / \mathrm{s}$ \\
\hline $\boldsymbol{G}_{\boldsymbol{t}}$ & 1 & $P_{t}$ & $15 \mathrm{dBm}$ \\
\hline $\boldsymbol{C W}_{\min }$ & 15 & $G_{r}$ & 1 \\
\hline $\boldsymbol{C W}_{\max }$ & 1023 & $R$ & $300 \mathrm{~m}$ \\
\hline $\boldsymbol{L}$ & 1024 byte & $r_{b}$ & $1 \mathrm{M}$ \\
\hline
\end{tabular}

posite directions along four-lane road pattern with a minimum average of $30 \mathrm{~m}$ space between any given pair of adjacent vehicles that are in the same lane. The Rayleigh model is used with the vehicles velocity randomly distributed in the range of the discrete set $V=\{(30+10 * k) m / s, \forall k \in[0,3]\}$ for the simulation of channel fading effect. Five data flows were set up at the rate of 100 packets $\mathrm{p} / \mathrm{s}$ as a default setting. The rest of the parameters used this simulation are shown in Table III.

\section{A. Results and Discussion}

We compare CARER performance against Simple Repetition (SR/ELR) based error recovery scheme [12], a scheme without network coding. In Fig.5(a), there is a significant improvement (over $20 \%$ in maximum) in packet loss recovery ability of CARER over SR scheme. This can be explained by the fact that conventional error recovery techniques based on retransmission of packets repeat each transmission separately thereby congesting the channel excessively as opposed to CARER which combines $n$ packets into one. In Fig.5(c), the PRP of SR scheme declined from 0.74 to 0.63 while CARER performance improved. This significant decline of SR's PRP is due to the fact that, though, retransmission-based loss recovery techniques increase the chances of recovering packets not received or correctly received, the repeated packets increase network overhead and after a given number of consecutive repeats may lead to excessive channel congestion and massive consumption of a substantial amount of the channel bandwidth thereby giving rise to excessive increase of network overhead and further loss of data transmissions due to QoS deterioration.

In both schemes, the percentage of packet collision probability (PCP) starts to increase considerably as packet generation probability (PGP) increases towards 1 (Fig.5(d)-(f)). This rapid increase in the PCP is caused by increased medium access contention due to high network saturation as the PGP increases. Similarly, Fig. 5(e)-(f) shows a gradual increase in the percentage of PCP for both CARER and SR protocol as the number of retransmission attempts increase. In any case, CARER outperforms the SR scheme due to reduced number of contending packets through network coding and the use of rebroadcast vehicle selection metric $\eta$.

From Fig.5(g)-(i), it is observed that, for both low and high packet generation rate (PGR), CARER can offer a performance advantage of multiple orders of magnitude against SR scheme in terms of Packet delivery rate (PDR). In Fig.5(h), the performance gap between CARER and SR gets even wider due to increase retransmission attempts. This is expected since every retransmission of the encoded message by CARER provides higher chances of data packets delivery given the increased data content through network coding.

In Fig.5(h)-(i), the performance of both protocols show a decline as the number of retransmission attempts climaxed at 7. However, the degree of performance degradation is more conspicuous in SR protocol. This is because retransmission-based error recovery proofs counter-productive after a certain number of attempts. The significant decline in PDR of SR protocol from 0.9 to 0.7 in Fig.5(i) is because the repeated packets resulted to increase network overhead because every one of the packets are transmitted separately as opposed to CARER which combines $n$ packets into one.

Fig. 6(a)-(c) clearly show that SR scheme suffers higher data delivery delay $\left(D^{3}\right)$ than CARER, and the performance gap steeply increases with increase in vehicular density and background noise $\left(N_{0}\right)$ levels. This is because: 1) the CARER uses the adjustment of performance parameters such as CWmin, CWmax, AIFSN, and PF to allow packets with higher QoS requirements to be accorded highest priority thereby resulting in a smaller $\mathrm{D}^{3}$ against SR protocol, and 2) CARER uses a rebroadcasting metric $\eta$ to successfully select the most suitable vehicle, which in turn, enables the selected node to only wait a minimum number of mini-slots to reply an RTB packet with a CTB packet thereby resulting in less delay as opposed to SR protocol. 
This article has been accepted for publication in a future issue of this journal, but has not been fully edited.

Content may change prior to final publication in an issue of the journal. To cite the paper please use the doi provided on the Digital Library page.

Similarly, in Fig. 6(d)-(f), as expected, CARER outperforms SR scheme over all workloads in multiple orders of magnitude due to: 1) the use of network coding to increase the content of each transmission, and 2) using a cross-layer approach empowered with $\eta$ to select only one rebroadcasting station, which not only can eliminate the inherent problem of broadcast storm associated with broadcast transmission but can alleviate the hidden station problem and increase the message transmission reliability as well. The system throughput of SR decreased almost by half compared to its result in Fig. 6(a). Again, this is because high number of retransmission attempts can lead to excessive network overhead and congestion, which can counter the performance in terms of achievable end-to-end system throughput for SR scheme.

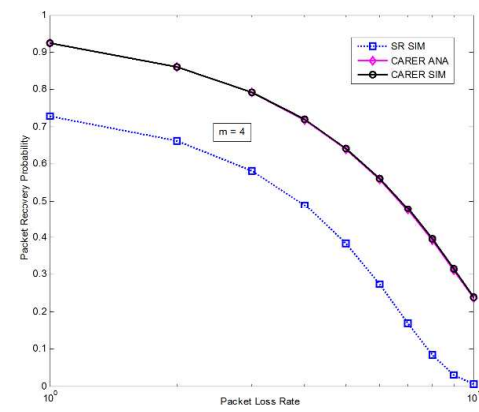

(a) $m=4$

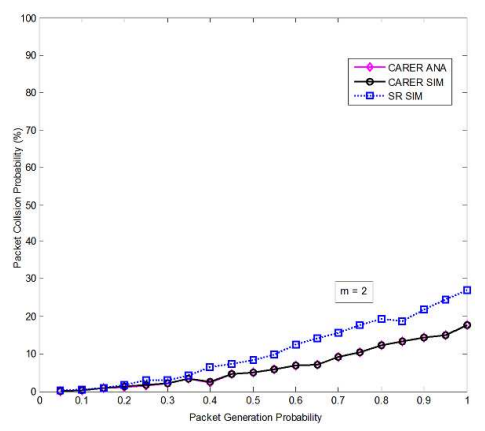

(d) $m=2$

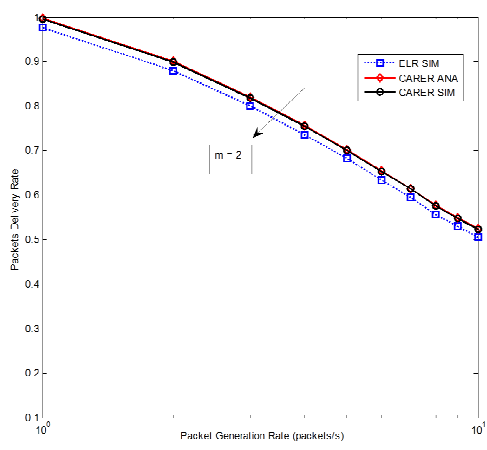

(g) $m=2$

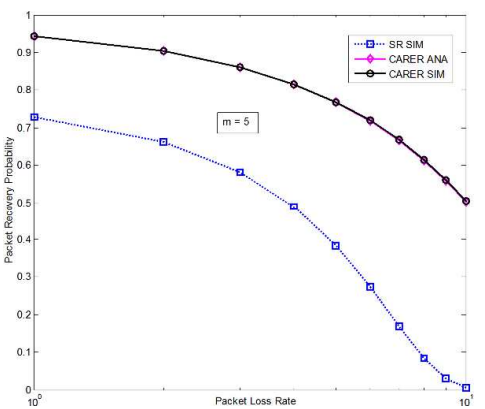

(b) $m=5$

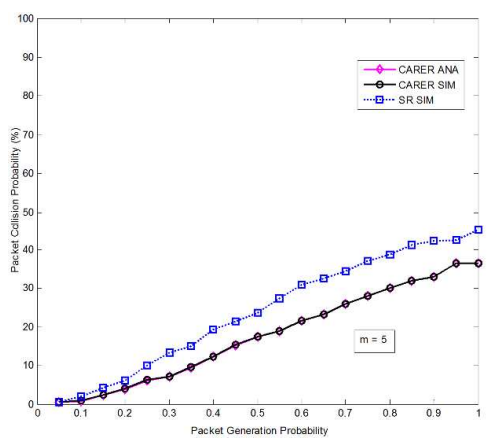

(e) $m=5$

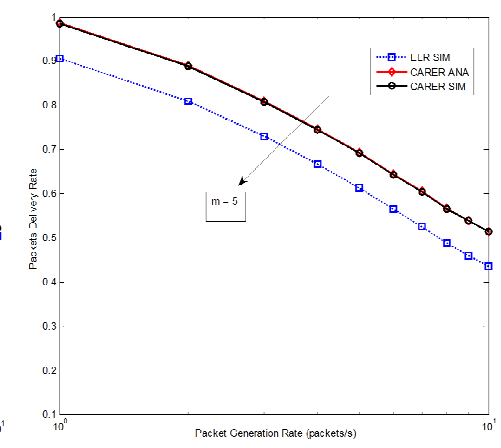

(h) $m=5$

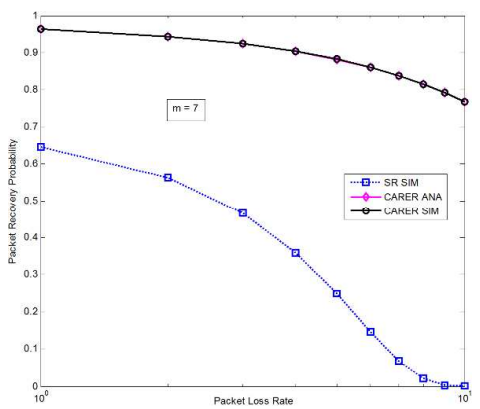

(c) $m=7$

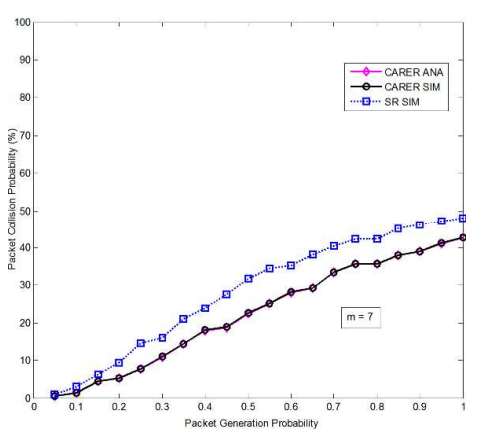

(f) $m=7$

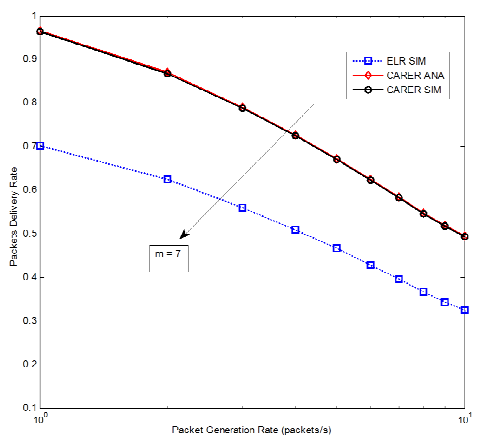

(i) $m=7$

Fig.5: Performance comparisons between the CARER and the SR scheme using (a)-(c) PRP, (d)-(f) percentage of PCP as a function of packet loss rate and PGP, respectively; (g)-(i) PDR as a function of packet generation rate (packets/s).

\section{CONCLUSIONS}

In this paper, we present an analytical model to achieve a timely and reliable packet broadcast in vehicular networks with the help of network coding concept. We developed a rebroadcasting metric $\eta$ which is used to determine and select the most suitably qualified candidate for rebroadcasting the encoded packets to enable the nodes outside the radio coverage of the source node to receive the encoded packets. Both the theoretical and simulation results show that the analytical model of the proposed CARER protocol is accurate in calculating the 
This article has been accepted for publication in a future issue of this journal, but has not been fully edited. Content may change prior to final publication in an issue of the journal. To cite the paper please use the doi provided on the Digital Library page.

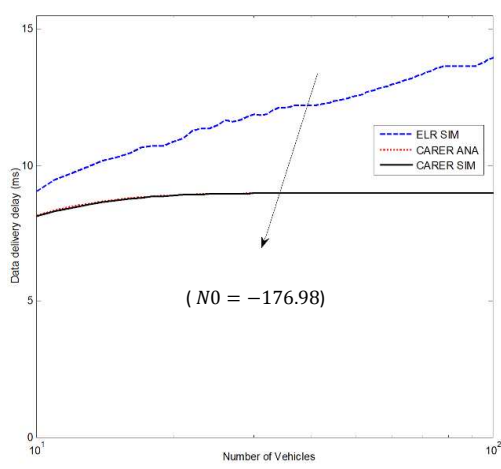

(a) $N_{0}=-176.98$

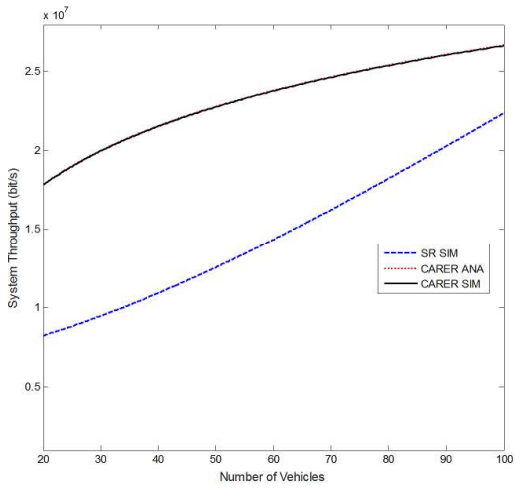

(d) $m=2$

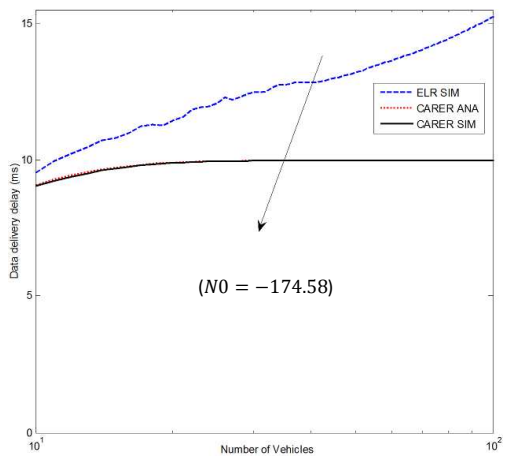

(b) $N_{0}=-174.58$

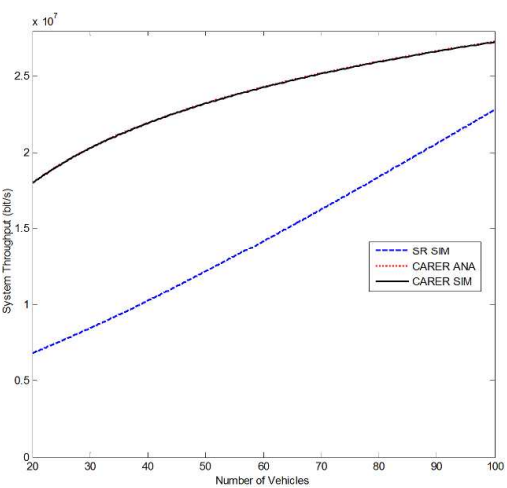

(e) $m=5$

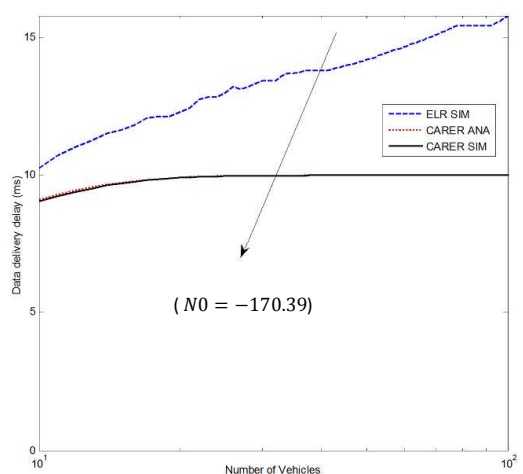

(c) $\left.N_{0}=-170.39\right)$

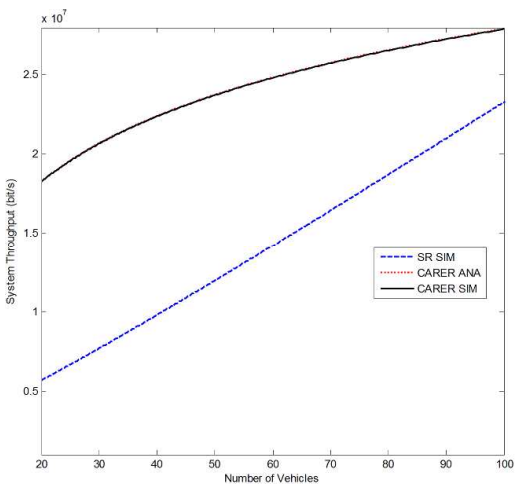

(f) $m=7$

Fig. 6. Performance comparisons between the CARER and the SR scheme using (a) - (c) data access delay, and (d) - (f) average system throughput as a function of number of vehicles (traffic density), respectively.

recovery of lost packet(s), reduction of PCP, high DDR, minimum $\mathrm{D}^{3}$, and high system throughput for both periodic status and safety (high priority mobile patient's bio-signal) packets. The performance evaluation of CARER protocol in urban environments which comprises of short road segments with intersections will form an interesting future work.

\section{REFERENCES}

[1] Eze C, E.; Sijing Zhang; Enjie Liu, "Improving Reliability of Message Broadcast over Internet of Vehicles (IoVs)," in 2015 IEEE International Conference on Computer and Information Technology; Ubiquitous Computing and Communications; Dependable, Autonomic and Secure Computing; Pervasive Intelligence and Computing (CIT/IUCC/DASC/PICOM), pp.2321-2328, 26-28 Oct. 2015

[2] Eze, Joy; Zhang, Sijing; Liu, Enjie; Chinedum, Elias E.; Yu, Hong Q., "Cognitive Radio Aided Internet of Vehicles (IoVs) for Improved Spectrum Resource Allocation," in 2015 IEEE International Conference on Computer and Information Technology; Ubiquitous Computing and Communications; Dependable, Autonomic and Secure Computing; Pervasive Intelligence and Computing (CIT/IUCC/DASC/PICOM), pp.2346-2352, 26-28 Oct. 2015

[3] The World Health Organization, "The World Health Report 2002 - Reducing Risks, Promoting Healthy Life." http://www.who.int/whr/2002/chapter4/en/index 7.html , 2002.

[4] Reported road casualties in Great Britain: main results 2014 by Department for Transport. Available online at: https:/www.gov.uk/government/uploads/system/uploads/attachment_data/file/438040/reported-road-casualties-ingreat-britain-main-results-2014-release.pdf

[5] Agbonkhese, O; Yisa, G.L; Agbonkhese, E.G; Akanbi, D.O; Aka, E.O; Mondigha, E.B. Road Traffic Accidents in Nigeria: Causes and Preventive Measures. IISTE Journal on Civil and Environmental Research, Vol.3, No.13, pp.9099, 2013

[6] Eze, E.C.; Sijing Zhang; Enjie Liu, "Vehicular ad hoc networks (VANETs): Current state, challenges, potentials and way forward," 20th International Conference on Automation and Computing (ICAC) 2014, pp.176-181, 12-13 Sept. 2014.

[7] D. Niyato, E. Hossain and S. Camorlinga, "Remote patient monitoring service using heterogeneous wireless access networks: architecture and optimization," in IEEE Journal on Selected Areas in Communications, vol. 27, no. 4, pp. 412-423, May 2009.

[8] Mrinmoy Barua, Xiaohui Liang, Rongxing Lu, and Xuemin (Sherman) Shen, "RCare: Extending Secure Health Care to Rural Area Using VANETs,” ACM Mobile Networks and Applications (MONET), vol. 19, no. 3, pp. 318-330, 2013. 
This article has been accepted for publication in a future issue of this journal, but has not been fully edited.

Content may change prior to final publication in an issue of the journal. To cite the paper please use the doi provided on the Digital Library page.

[9] Yuanguo Bi; Cai, L.X.; Xuemin Shen; Hai Zhao, "Efficient and Reliable Broadcast in Intervehicle Communication Networks: A Cross-Layer Approach," IEEE Transactions on Vehicular Technology, vol.59, no.5, pp.2404-2417, Jun 2010

[10] F. Farnoud and S. Valaee, "Repetition-based broadcast in vehicular ad hoc networks in rician channel with capture," in IEEE INFOCOM Workshops, pp. 1-6, 2008

[11] L. Yang, G. Jinhua, and W. Ying, "Piggyback cooperative repetition for reliable broadcasting of safety messages in vanets," in 6th IEEE Consumer Communications and Networking Conference, CCNC, 2009

[12] R. Ahlswede, N. Cai, S.Y.R. Li, and R.W. Yeung, "Network Information Flow," IEEE Transactions on Information Theory, vol. 46, no. 4, pp.1204-1216, July 2000

[13] P. Yang, M. Hanneghan, J. Qi, Z. Deng, D. Fan and F. Dong, "Improving the Validity of Lifelogging Physical Activity Measures in an Internet of Things Environment", pp. 2309-2314, the 2015 IEEE International Conference on Pervasive Intelligence and Computing, Liverpool, UK, October, 2015.

[14] J. Qi, P. Yang, D. Fan, Z. Deng, “A survey of Physical Activity Monitoring and Assessment Using Internet of Things technology", the 2015 IEEE International Conference on Pervasive Intelligence and Computing, Liverpool, UK, pp. 2353-2358, October, 2015.

[15] Z. Deng, P. Yang, Y. Zhao, X. Zhao and F. Dong, "Life-logging data aggregation solution for interdisciplinary healthcare research and collaboration", the 2015 IEEE International Conference on Pervasive Intelligence and Computing, pp. 2315-2320, Liverpool, UK, October, 2015.

[16] E. G. Spanakis, D. Kafetzopoulos, P. Yang, K. Marias, Z. Deng, M. Tsiknakis, V. Sakkalis and F. Dong, "MyHealthAvatar: Personalised and empowerment health services through Internet of Things technologies", the 2014 EAI $4^{\text {th }}$ International Conference on Wireless Mobile Communication and Healthcare, Athens, Greece, November, 2014.

[17] V. Codreanu, D. Williams, J. Roerdink, P. Yang, B.Q.Liu, F. Dong, "GPU-ASIFT: A Fast Fully AffineInvariant Feature Extraction”, the 2013 High Performance Computing \& Simulation Conference, Helsinki, Finland, July, 2013.

[18] B. Q. Liu, A.C. Telea, J. Roerdink, G. J. Clapworthy, D. Williams, P. Yang, F. Dong, V. Codreanu, A. Chiarini, "Parallel Centerline Extraction on GPU for Virtual Endoscopy", Computer \& Graphics, Mar, 2014.

[19] U. Varshney and S. Sneha, "Patient monitoring using ad hoc wireless networks: reliability and power management," in IEEE Communications Magazine, vol. 44, no. 4, pp. 49-55, April 2006.

[20] J. K. Pollard, S. Rohman and M. E. Fry, "A Web-based mobile medical monitoring system," International Workshop on Intelligent Data Acquisition and Advanced Computing Systems: Technology and Applications, Crimea, pp. 32-35, 1-4 Jul 2001.

[21] G. G. Mendoza and B. Q. Tran, "In-home wireless monitoring of physiological data for heart failure patients," 24th Annual Conference and the Annual Fall Meeting of the Biomedical Engineering Society EMBS/BMES Conference on Engineering in Medicine and Biology, vol.3, pp. 1849-1850, Oct. 2002

[22] M. D'Souza, M. Ros and A. Postula, "Wireless Medical Information System Network for Patient ECG Monitoring," 9th EUROMICRO Conference on Digital System Design (DSD'06), Dubrovnik, pp. 617-624, 2006.

[23] T. Suzuki and M. Doi, "LifeMinder: An Evidence-Based Wearable Healthcare Assistant," in Proceedings of ACM CHI Conference, pp. 127-128, Mar.-Apr. 2001.

[24] M. Modarreszadeh and R. N. Schmidt, "Wireless, 32-channel, EEG and epilepsy monitoring system," Engineering in Medicine and Biology Society. Proceedings of the 19th Annual International Conference of the IEEE, Chicago, IL, vol.3, pp. 1157-1160, 30 Oct-2 Nov 1997.

[25] U. Varshney and S. Sneha, "Wireless patient monitoring: reliability and power management," 2nd International Conference on Broadband Networks, Boston, MA, Vol. 2, pp. 1034-1040, Oct. 2005.

[26] Li, S.-Y. R.; Ning Cai; Yeung, R.W., "On theory of linear network coding," 2005. ISIT 2005 Proceedings. International Symposium on Information Theory, Adelaide, SA, pp.273-277, 4-9 Sept. 2005

[27] D. S. Lun, N. Ratnakar, R. Koetter, M. M'edard, E. Ahmed, and H. Lee. Achieving Minimum-Cost Multicast: A Decentralized Approach Based on Network Coding. In IEEE INFOCOM, 2005

[28] Dong Nguyen; Tran, T.; Thinh Nguyen; Bose, B., "Wireless Broadcast Using Network Coding,", IEEE Transactions on Vehicular Technology, vol.58, no.2, pp.914,925, Feb. 2009.

[29] Zhe Wang; Hassan, M., "Network Coded Repetition: A Method to Recover Lost Packets in Vehicular Communications," in 2011 IEEE International Conference on Communications, (ICC), pp.1-6, June 2011.

[30] Eze, E.C.; Sijing Zhang; Liu, E., "Estimation of collision probability in a saturated vehicular Ad-Hoc networks," in 2015 IEEE Fourth International Conference on Future Generation Communication Technology (FGCT), pp.1-7, 29-31 July 2015

[31] Eze, Elias C.; Sijing Zhang; Enjie Liu, Eze, Joy C., "Advances in Vehicular Ad-hoc Networks (VANETs): Challenges and Road-map for Future Development," International Journal of Automation and Computing, Vol.13, No.1, pp. 1-18, 2016

[32] J. G. Proakis, Digital Communications, 4th ed. New York: McGraw-Hill, 2000.

[33] Roberts, J.A., "Packet error rates for DPSK and differentially encoded coherent BPSK," IEEE Transactions on Communications, vol.42, no.234, pp.1441-1444, Feb/Mar/Apr 1994.

[34] [Online]. Available: http://www.isi.edu/nsnam/ns/ 